\title{
Marker-assisted selection and yield component assessment of spring wheat plants derived from crosses between spring and winter wheat cultivars
}

\author{
A.I. Stasyuk, I.N. Leonova, E.A. Salina \\ Institute of Cytology and Genetics, SB RAS, Novosibirsk, Russia
}

DOI 10.18699/ICG-PlantGen2019-62

(c) Autors, 2019

*e-mail: stasyuk@bionet.nsc.ru

\begin{abstract}
The effectiveness of the use of marker-assisted selection for identification of spring growth-habit genotypes derived from crosses between soft winter wheat cultivars and the spring wheat introgression line 21-4 carrying the Ae. speltoides LrAsp5 gene for resistance to leaf rust has been demonstrated. The duration of the period from sprouting to heading has been shown to vary across the hybrids in both directions relative to the parental line, even though the allelic compositions of the $V R N-1$ genes were identical. The presence of foreign genetic material has no effect on heading date. Plants with $\operatorname{LrAsp} 5$ are highly resistant to leaf rust. The expression of the quantitative traits follows an individual pattern in each line, irrespective of the presence of alien translocations.

Key words: spring soft wheat; $V R N-1$ genes; $L r$ genes; marker-assisted selection; yieldrelated traits.
\end{abstract}

\section{Introduction}

Spring soft wheat is the main crop in West Siberia, having more than $50 \%$ of the land area under crops and pulse crops. The first priority of spring soft wheat breeding is the development of high-yield cultivars with resistance to biotic and abiotic environmental factors. To this end, the breeders search for, find and use donors of important traits in crosses. The good choice is soft winter wheat, which has advantages over spring wheat in terms of yield. Identification of homozygous spring wheat plants resulting from crosses between winter and spring wheats requires large timescales. The use of molecular markers can make the search for genotypes of interest less time- and labor-consuming. In our study, the winter wheat cultivars 'Biyskaya ozimaya', 'Filatovka', 'Novosibirskaya 3' and 'Novosibirskaya 40' adapted to West Siberia were used as the maternal forms. The spring wheat introgression line 21-4 with a translocation on the long arm of Ae. Speltoides chromosome 5B carrying the LrAsp5 gene was used the pollinator. The aim of our work was to identify, using molecular markers, soft wheat plants with a spring growth habit in the hybrid population and to assess the quality of their agronomically important traits.

\section{Materials and methods}

The material used were hybrid plants derived from crosses between each of the soft winter wheat cultivars 'Biyskaya ozimaya', 'Filatovka', 'Novosibirskaya 3' and 'Novosibirskaya 40' and the spring wheat introgression line 21-4 (T. aestivum/Ae. speltoides) carrying the LrAsp 5 locus for resistance to leaf rust. We were searching the $\mathrm{F}_{2}$ population for spring and winter growth-habit plants using allele-specific markers for the Vrn-A1, Vrn-B1 and Vrn-D1 genes as described in a work by Likhenko et al. (2015). To identify plants carrying the LrAsp 5 gene from Ae. speltoides, the Pr1 and Pr5 primers were used, their structure and PCR conditions for them as described in a work by Leonova et al. (2017). Agriculturally important traits were assessed in $\mathrm{F}_{3}$ plants on an experimental field (Michurinskiy Settlement, Novosibirsk Region) in 2018. The parental spring wheat line 21-4 was used as the control. The traits assessed were: number of days from sprouting to heading, resistance to leaf rust, grain number per spike, grain weight per spike and 1000-grain weight. Resistance to leaf rust was assessed using the $0-4$ scale by Mains and Jackson (Mains, Jackson, 1926). Differences between the mean values of two samples were tested for significance using Student's $t$-criterion.

\section{Results and discussion}

Crosses between each of the winter soft wheat cultivars 'Biyskaya ozimaya', 'Filatovka', 'Novosibirskaya 3' and 'Novosibirskaya 40' and wheat introgression line 21-4 resulted in 303 hybrid plants, from among which 90 homozygous spring-habit $\mathrm{F}_{2}$ plants were identified using molecular markers linked to the $V R N-1$ genes. Of them, 24 had translocations from Ae. speltoides, detected with the use of the molecular markers Pr1 and Pr5.

Agronomically valuable traits were analyzed in three 'Biyskaya ozimaya' $\times$ 21-4 hybrid plants, of which two had Ae. speltoides translocations, 18 'Filatovka' $\times 21-4$ hybrid plants, of which five had Ae. speltoides translocations, 15 'Novosibirskaya 3' $\times 21-4$ hybrid plants, of which four had Ae. speltoides translocations and 28 'Novosibirskaya 40' $\times$ 21-4 hybrid plants, of which ten had Ae. speltoides translocations. The parental spring wheat line 21-4 was used as the control.

Heading date was substantially influenced by the allelic composition of the $V R N-1$ genes controlling growth habit (Stelmakh, 1993; Trevaskis et al., 2007). Our analysis of these genes showed that all spring growth-habit progeny of the crosses between each of the soft winter wheat cultivars and the spring line had the same allelic combination as had the parental line 21-4 - Vrn-Ala, vrn-B1, and vrn-D1. In line 21-4, the number of days from sprouting to heading was 41.1 . In the 'Biyskaya ozimaya' $\times 21-4$ progeny, three out of 13 lines 
Table 1

Number of days from sprouting to heading and leaf rust resistance in lines with LrAsp5

\begin{tabular}{|c|c|c|c|c|}
\hline \multirow{2}{*}{ Cross } & \multirow{2}{*}{ Line } & \multirow{2}{*}{$\begin{array}{l}\text { Sprouting to heading, days } \\
x \pm s x\end{array}$} & \multicolumn{2}{|c|}{ Leaf rust resistance } \\
\hline & & & Infection type 1 & Percent leaf area infected \\
\hline Line 21-4 & Control & $41.1 \pm 0.2$ & 1 & 5 \\
\hline \multirow[t]{2}{*}{ Biyskaya ozimaya × 21-4 } & $77-7$ & $39.4 \pm 0.2^{*}$ & 1 & 10 \\
\hline & $77-10$ & $42.3 \pm 0.3^{*}$ & 0 & 0 \\
\hline \multirow[t]{5}{*}{ Filatovka $\times 21-4$} & $83-3$ & $39.4 \pm 0.2^{*}$ & 0 & 0 \\
\hline & $83-10$ & $41.4 \pm 0.2$ & 0 & 0 \\
\hline & $83-11$ & $41.4 \pm 0.2$ & 1 & 15 \\
\hline & $84-14$ & $41.1 \pm 0.3$ & 0 & 0 \\
\hline & $87-8$ & $40.1 \pm 0.4$ & 1 & 5 \\
\hline \multirow[t]{4}{*}{ Novosibirskaya $3 \times 21-4$} & $31-8$ & $38.5 \pm 0.1^{*}$ & 2 & 20 \\
\hline & $31-14$ & $38.2 \pm 0.3^{*}$ & 2 & 30 \\
\hline & $32-10$ & $39.3 \pm 0.3^{*}$ & 1 & 20 \\
\hline & $34-12$ & $40.3 \pm 0.2^{*}$ & 1 & 10 \\
\hline \multirow[t]{10}{*}{ Novosibirskaya $40 \times 21-4$} & $53-1$ & $41.2 \pm 0.3$ & 2 & 50 \\
\hline & $53-10$ & $37.8 \pm 0.3^{*}$ & 2 & 40 \\
\hline & $54-6$ & $42.7 \pm 0.4^{*}$ & 0 & 0 \\
\hline & $54-7$ & $41.0 \pm 0.2$ & 0 & 0 \\
\hline & $54-10$ & $39.8 \pm 0.3^{*}$ & 2 & 30 \\
\hline & $54-14$ & $42.3 \pm 0.2^{*}$ & 2 & 30 \\
\hline & $56-8$ & $43.3 \pm 0.4^{*}$ & 0 & 0 \\
\hline & $57-5$ & $40.4 \pm 0.4$ & 1 & 10 \\
\hline & $57-8$ & $39.9 \pm 0.3^{*}$ & 0 & 0 \\
\hline & $57-11$ & $39.4 \pm 0.2^{*}$ & 2 & 30 \\
\hline
\end{tabular}

* Differences between the hybrid lines and the parental line are significant at $p<0.05$.

${ }^{1}$ On the 0-4 scale after Mains and Jackson.

were later in ear emergence than line 21-4. Line 77-10 with an alien translocation was the latest - it was 1.2 days later than the control (Table 1). Other three lines were earlier than the control. Line 77-7 with a translocation was the earliest - it was 1.7 days earlier than the parental line. No other line was different from line 21-4. In the 'Filatovka' $\times$ 21-4 progeny, only line $85-1$ was later than the parental line -2.9 days later. Five lines were earlier than the control, with line 86-2 being the earliest - it was 2.8 days earlier. All the other lines, including those with translocations, in this hybrid population were similar to the control. In the 'Novosibirskaya 3' $\times 21-4$ hybrid population, only two lines, $31-12$ and $32-7$, had the same heading date as the parental line 21-4. All the other lines in this hybrid population were earlier than their spring growth-habit parent. Line 35-12 was the earliest - it was 4.3 days earlier than the control. In the 'Novosibirskaya 40' $\times 21-4$ progeny, four lines were later than, 12 were earlier than and 12 were not different from the parental line 21-4. Line 56-8 with a translocation was the latest - it was 2.2 days later than the control. Line 53-10 with a translocation was the earliest - it was 3.3 days earlier than the control.

Heading dates in the lines with foreign genetic material in their genomes were shown to be earlier than, equal to or later than that in the control line 21-4. The same was true of the lines without alien introgressions. It can therefore be concluded that the translocation from Ae. speltoides has no effect on heading date in soft wheat. It had previously been demonstrated that hybrid plants derived from crosses between winter and spring wheats were later in ear emergence than the spring growth-habit parent, even though the allelic compositions of the $V R N-1$ genes were identical (Stasyuk et al., 2017). The hybrid plants resulting from the crosses between each of the winter cultivars and line 21-4 had the same allelic composition of the $V R N-1$ genes as had the parental line, but their heading dates varied in both directions relative to the control. It is likely that the differences in heading date are explained by factors other than $V R N-1$.

Scoring the severity of leaf rust showed that all the lines that had translocations from Ae. speltoides were immune or slightly susceptible (Table 1). Lines without LrAsp5 were highly susceptible and had infection type 4 on the $0-4$ scale by Mains and Jackson, with pustules covering up to $100 \%$ of the leaf area.

Analysis of yield components showed that, in the 'Biyskaya ozimaya' $\times$ 21-4 progeny, grain number per main spike was similar to that in the parental line in almost all lines, except for 
line 74-8 without translocations and line 77-7 with a translocation from Ae. speltoides, which were inferior to the control. In the 'Filatovka' $\times 21-4$ progeny, ten lines were equal to line 21-4, and eight lines, including line 83-10 with a translocation, were inferior to the control. In the 'Novosibirskaya 3' $\times 21-4$ progeny, line 36-3 without translocations was superior and five lines, including two with translocations, were inferior to the control. In the 'Novosibirskaya $40^{\prime} \times 21-4$ progeny, five lines, of which two had foreign genetic material, were inferior to the control. No other line was different from the spring growth-habit parent.

Grain weight per spike in the 'Biyskaya ozimaya' $\times 21-4$ progeny was not significantly different from the control, except for line 74-8, which was inferior. An line with an Ae. speltoides introgression, 83-3, which was superior to the parental line, was identified among the 'Filatovka' $\times$ 21-4 hybrids. Seven lines, of which one had a translocation, were inferior to the control, while the others were similar to the spring growth-habit parent. Two 'Novosibirskaya 3' $\times$ 21-4 hybrid lines without translocations were significantly superior to the control, and four were inferior in terms of grain weight per spike. The other nine lines in that progeny were not different from the parent. In the 'Novosibirskaya 40' $\times 21-4$ progeny, seven lines, including three with translocations, were inferior to the parental line, while the others were not different from the control.

1000-grain weight in line 75-13 without translocations and line 77-7 with a translocation from cross 'Biyskaya ozimaya' $\times$ 21-4 was significantly higher than in the control, three lines were inferior and the other eight were similar to the control in terms of this measure. In the 'Filatovka' $\times 21-4$ progeny, four lines were superior and four were inferior to the control. In either case, one of the lines had a translocation. The other lines in that population were not significantly different from the control. In the 'Novosibirskaya 3' $\times 21-4$ progeny, five lines, including one with a translocation, were superior, three were inferior, and seven were similar to the control. In the 'Novosibirskaya 40' $\times 21-4$ progeny, four lines, of which two had translocations, were superior to the control. Three lines, of which one had a translocation, were significantly inferior and 21 were significantly similar to the control.

The assessment of the quantitative traits showed that the lines with translocations from Ae. speltoides were superior, equal or inferior to the control in terms of those traits. It can therefore be concluded that the expression of the quantitative traits in each line follows an individual pattern, irrespective of the presence of alien translocations. Based on the assessment of yield components, lines 75-13, 83-3, 84-10, 31-15, 35-3, $36-3,36-6,54-2,56-7,57-8$, and 57-11 were significantly superior to the parental line 21-4 in terms of 1000-grain weight or grain number per main stem spike and grain weight per main stem spike. These lines can be recommended for use in breeding work.

\section{Conclusions}

Our results show the effectiveness of marker-assisted selection, with which we identified among $\mathrm{F}_{2}$ plants those that had a spring growth habit and carried a translocation with the LrAsp5 gene from Ae. speltoides. A field experiment demonstrated that plants with translocations were highly resistant to the West Siberian population of Puccinia triticina Erikss., the causative agent of leaf rust. Variation in heading date is probably not only due to the $V R N-1$, genes, but also due to the genetic background of the winter wheat varieties used. The expression of the quantitative traits follows an individual pattern in each line, irrespective of the presence of alien translocations.

\section{References}

Likhenko I.E., Stasyuk A.I., Shcherban'A.B., Zyryanova A.F., Likhenko N.I., Salina E.A. Study of allelic composition of Vrn-1 and $P p d-1$ genes in early-ripening and middle-early varieties of spring soft wheat in Siberia. Rus. J. Genet.: Applied Research. 2015;5(3): 198-207.

Leonova I.N., Stasyuk A.I., Skolotneva E.S., Salina E.A. Enhancement of leaf rust resistance of Siberian winter wheat varieties by markerassisted selection. Cereal Research Communications. 2017;45(4): 621-632.

Mains E.B., Jackson H.S. Physiological specialization in the leaf rust of wheat, Puccinia triticina Erikss. Phytopathol. 1926;16:89-120.

Stelmakh A.F. Genetic effects of Vrn genes on heading date and agronomic traits in bread wheat. Euphytica. 1993;65:53-60.

Trevaskis B., Hemming M.N., Dennis E.S., Peacock W.J. The molecular basis of vernalization-induced flowering in cereals. Trends Plant Sci. 2007;12(8):352-357.

Stasyuk A.I., Leonova I.N., Salina E.A. Variability of agronomically important traits in spring wheat hybrids obtained by marker-assisted selection from crosses of winter wheat with spring wheat donors of resistance genes. Sel'skokhozyaistvennaya Biologiya $=$ Agricultural Biol. 2017;52(3):526-534.

Acknowledgements. This work was supported by grant No. 16-1600011 from the Russian Science Foundation.

Conflict of interest. The authors declare no conflict of interest. 